\title{
Carnets
}

Revue électronique d'études françaises de l'APEF

Deuxième série - 9 | 2017

Reconnaissances et légitimité en français

\section{Le corps de l'écrivain, instrument et enjeu de reconnaissance}

\section{Paul Dirkx}

\section{(2) OpenEdition}

Journals

\section{Édition électronique}

URL : http://journals.openedition.org/carnets/2024

DOI : 10.4000/carnets.2024

ISSN : 1646-7698

Éditeur

APEF

\section{Référence électronique}

Paul Dirkx, «Le corps de l'écrivain, instrument et enjeu de reconnaissance », Carnets [En ligne], Deuxième série - 9 | 2017, mis en ligne le 31 janvier 2017, consulté le 01 mai 2019. URL : http:// journals.openedition.org/carnets/2024; DOI : 10.4000/carnets.2024

Ce document a été généré automatiquement le 1 mai 2019.

\section{(c) (i) (8)}

Carnets est mis à disposition selon les termes de la licence Creative Commons - Atribution - Pas d'utilisation commerciale 4.0 International. 


\title{
Le corps de l'écrivain, instrument et enjeu de reconnaissance
}

\author{
Paul Dirkx
}

1 Cette étude propose un ensemble d'hypothèses concernant les rapports entre la reconnaissance littéraire et les corps des écrivains. Une première partie tentera d'articuler les notions de reconnaissance, de notoriété, de légitimité, etc., que de nombreux travaux perçoivent tantôt comme discordantes, tantôt comme partiellement redondantes. Nous tenterons de cerner ce qui les rassemble et ce sur quoi elles divergent, en nous inspirant de la théorie des champs. Ce n'est qu'après ce nécessaire éclaircissement que nous pourrons traiter de ce qui paraît être au fondement des mécanismes de reconnaissance littéraire : une autonomie elle-même basée sur une forme spécifiquement littéraire de la croyance. Cela amènera ensuite à convoquer la notion d'incorporation et à considérer les écrivains dans leurs dimensions inséparablement somatiques et symboliques, « sociobiologiques » (Elias, 2015). Enfin, certaines hypothèses seront nuancées à travers deux exemples d'écrivains qui ont mis en texte leur propre corps d'écrivain : Claude Simon et Christian Dotremont.

\section{Jeux et enjeux de la reconnaissance}

2 La plupart des travaux consacrés directement ou indirectement à l'évaluation sociale des œuvres et de leurs auteurs omettent de spécifier ce qu'ils entendent par " reconnaissance », «notoriété », « légitimité », « légitimation », « consécration », « canonisation", «classicisation » ou encore "panthéonisation ». Souvent, il s'agit d'un oubli ; parfois, cette omission est voulue, le chercheur estimant ne pas avoir à apporter des précisions qui n'ont pas cours sur le terrain littéraire lui-même, où ces mots sont rarement définis et se chevauchent allègrement.

3 Mais s'il est vrai qu'il faut se méfier des «usages, parfois peu contrôlés ${ }^{1}$ », de mots devenus par trop familiers, si l'on a intérêt à ne pas confondre concepts scientifiques et mots de la tribu, notions et prénotions (Durkheim, 1997 : 31), l'étude de la littérature n'en a pas moins besoin d'outils opératoires permettant d'appréhender le fonctionnement 
axiologique de cette même tribu, fût-ce pour conclure à la complexité la plus rebutante. En s'inspirant de la théorie des champs, il devient possible, nous semble-t-il, de départager assez clairement ce qui relève de la reconnaissance de ce qui relève de la notoriété ou de la légitimité, etc. en tant que concepts à (re)définir, et ce en adéquation avec la réalité, certes mouvante, du terrain. Ce travail aux allures un peu austères est un préalable indispensable à notre propos.

Il convient de commencer par reconnaissance, dans la mesure où ce concept correspond au stade élémentaire du processus de mise en valeur, à savoir une sorte d'appel à la vie sociale adressé à un agent au sein d'un groupe, un acte de naissance sociale ou, si l'on préfère, un acte de renaissance. La reconnaissance est une identification valorisante de l'agent par d'autres agents habilités (légitimés, cf. infra) à cet effet (Bourdieu, 1979: 367-377). Cette identification fait de lui un membre à part entière du groupe, c'est-à-dire réputé avoir acquis les catégories de perception propres à lui faire reconnaitre le groupe, autrement dit propres à lui faire méconnaître, donc reconnaitre spontanément le pouvoir, pourtant toujours arbitraire, de ceux qui le reconnaissent au nom de l'idée qu'ils se font du groupe ${ }^{2}$. La reconnaissance désigne l'agent comme étant doté des «bonnes » compétences cognitives et pratiques qui, issues du groupe et le cautionnant, sont appelées à lui procurer un minimum de biens gratifiants censés le lier une fois pour toutes au groupe, ce que Pierre Bourdieu appelle l'obtention d'un «capital spécifique de reconnaissance » ou « capital symbolique » (Bourdieu, 1994: 187). Il convient encore de faire la part, non pas catégorique mais dialectique, entre reconnaissance et notoriété: par exemple, dans le sous-champ de production littéraire pour les pairs, les agents tendent à aspirer à la reconnaissance "purement » littéraire de leurs pairs et à l'accroissement endogène de leur capital symbolique, tandis que le désir d'une notoriété dépassant les limites du champ littéraire caractérise plutôt les agents du sous-champ de grande production (Bourdieu, 1994: 75) et implique d'abord leur capital exogène, économique et social.

5 La légitimité est la reconnaissance devenue comme "naturellement», c'est-à-dire arbitrairement universelle dans les limites du groupe (Bourdieu, 1963: 107-138; Bourdieu, $1994: 241$ ). Elle dote l'agent d'un pouvoir de représentation du groupe tout entier. On peut dire, s'agissant de littérature, que tout écrivain légitime est un écrivain reconnu, mais que l'inverse n'est pas vrai. La légitimité est le produit d'un travail de légitimation, d'une montée en puissance de la reconnaissance et de la production de capital symbolique qui fait basculer l'agent bénéficiaire du côté des agents dominants, ayant donc le pouvoir de diffuser dans le champ leur conception de ce qui s'y fait et qui, faisant ainsi autorité (auctoritas), sont légitimés à se reconnaître et à reconnaître à d'autres le statut d'auteur (auctor). Comme l'écrivait en 1963 Pierre Bourdieu, soucieux de faire de « légitimité » un « concept opératoire » en sciences sociales, ce terme désigne la «prétention à être universellement reconn[u]» (Bourdieu, 1963: 135) - "prétention», car la légitimité ne fait jamais l'unanimité délibérée dans le champ : "Est légitime une institution, ou une action, ou un usage qui est dominant et méconnu comme tel, c'est-à-dire tacitement reconnu ${ }^{3} »$ (Bourdieu, 2002: 110). La légitimation repose ainsi sur la méconnaissance de ce qu'elle est dans les faits: un coup de force au regard de sa prétendue universalité. Elle a pour forme "par excellence» (Bourdieu, 1997: 94), inculquée notamment par l'institution scolaire qui intervient à partir de ce stade, la rationalisation ou, mieux encore, l'universalisation. Pourtant, elle légitime ce qui objectivement ne peut l'être, à savoir un individu ou un modèle conformes au point de 
vue partiel et partial d'une instance particulière, non universelle, et condamnée à ce titre à l'imposition de son point de vue et donc à une conduite tout sauf rationnelle. Alors que la reconnaissance est plutôt un vecteur d'intégration, la légitimation ne l'est plus qu'en partie, car elle appelle son contraire et frappe d'illégitimité ce qui se heurte à ses critères d'évaluation.

6 Enfin, ce processus peut pousser la violence méconnue comme telle, symbolique, jusqu'à la consécration, soit atteindre un seuil en principe irréversible à partir duquel l'agent légitimé non seulement illustre, mais en vient à incarner le nomos, le fondement ultime, sacré, la raison d'être du système social concerné, par exemple littéraire. De l'écrivain exemplaire qu'il était, auteur d'un certain nombre d'œuvres reconnues comme illustrant tel ou tel modèle d'écriture, cet agent devient alors écrivain tout court, véritable dignitaire de "la littérature", non plus simple représentant légitime, mais haut représentant plus ou moins charismatique de cet art, pourvu d'un pouvoir instituant et donc habilité à son tour à consacrer, c'est-à-dire "à sanctionner et à sanctifier " (Bourdieu, 1982 : 124) la frontière entre écrivains légitimes et illégitimes (et, a fortiori, entre écrivains et non-écrivains). Ainsi, cet agent devient membre d'« une élite consacrée, c'est-à-dire non seulement distincte, séparée, mais aussi reconnue et se reconnaissant comme digne de l'être » (Bourdieu, 1989 : 140). On voit ainsi que la consécration déborde la sphère littéraire et irradie dans toute la société ou, plus exactement, dans tout le champ du pouvoir par l'intermédiaire de ses relais pédagogiques, médiatiques et autres.

7 Une fois que l'on accède aux sphères éthérées du sacré et de la consécration, la valorisation des textes et des auteurs s'apparente à une sacralisation, et le vocabulaire usité tient toujours plus de la métaphore religieuse (Denis, 2010 : § 4-8; Robert, 2002 : 70-72) - remarque qui n'a rien d'anodin, le champ religieux jouant, on y reviendra, un rôle fondateur dans la genèse et la structure du champ littéraire (Bourdieu, 1971: 295-334; Bourdieu, 1992). La formule " consécration littéraire » a longtemps correspondu en anglais à "literary canonisation », expression qui est ensuite passée dans la langue française $e^{4}$ et à laquelle " consécration littéraire " se voit souvent assimilée. La canonisation est l'inscription d'un nom et d'une œuvre au " canon » des grands écrivains « classiques " d'une littérature, autrement dit encore à son "panthéon ». Captée par les politiques identitaires des États menées à travers les institutions scolaires ${ }^{5}$, culturelles et médiatiques à des fins de "patrimonialisation", la canonisation est une classicisation (assimilée pour certains écrivains à une panthéonisation), vocable que l'usage français tend d'ailleurs toujours à lui préférer. Elle constitue un palier supplémentaire dans la marche vers la déification (qu'exprime "panthéonisation ») ou vers la fétichisation, ces deux dernières notions étant exclues, car trahissant le caractère objectivement délirant mais bien fondé, pour paraphraser Émile Durkheim parlant de la religion (Durkheim, 2008 : 324), de tout le processus de reconnaissance littéraire.

\section{Reconnaissance, autonomie et croyance littéraires}

8 Seule une minorité d'écrivains parcourent toutes les étapes de cette ascension ardue qui, semée d'embûches, est tout sauf un long fleuve tranquille. Elle se caractérise néanmoins par une cohérence non négligeable, raison pour laquelle il serait hasardeux de l'étudier dans son ensemble de manière indifférenciée ou, à l'inverse, d'en traiter l'un ou l'autre moment de manière isolée. Cette cohérence tient tout d'abord au fait que, quel que soit le volume de capital symbolique accumulé au fil du temps, la reconnaissance en demeure le 
substrat, en tant qu'elle «offre aux humains ce dont ils sont le plus totalement dépourvus: une justification d'exister" (Bourdieu, 1997: 282). Cette phrase aux apparences un peu spéculatives prend tout son sens, lorsqu'on songe aux trajectoires d'écrivains francophones nés sous les soleils des dépendances, pour faire référence au premier roman de l'Ivoirien Ahmadou Kourouma (Kourouma, 1968), romancier aujourd'hui classicisé ou en voie de l'être, mais qui, parti de rien, a dû, comme tous ses confrères subsahariens, s'imposer au regard occidental, encore très peu enclin à prêter attention à cette partie de l'humanité écrivante.

9 Ensuite, à mesure que l'on passe de la simple reconnaissance à la légitimité, puis à la consécration, la valeur cumulative des marques de distinction est proportionnelle à leur retentissement dans l'espace littéraire, puis social ${ }^{6}$. Et du fait que leur propagation plus grande implique des relais (médiatiques, critiques, scolaires, etc.) plus complexes et une violence symbolique plus intense et à la fois plus inaperçue, leur valeur augmente aussi avec la dénégation de leur fondement arbitraire (si la méconnaissance est la condition première de la reconnaissance en général, la dénégation apparait comme celle de la consécration en particulier). On s'aperçoit donc que la logique de la reconnaissance ne se révèle pleinement que si est révélé le système de domination dont elle tire sa force, à commencer par la violence symbolique qui conditionne ce système.

Car que signifie au juste domination? La domination est l'effet d'une suggestion réussie, d'une accréditation durable d'un schème de pensée et d'action présenté comme surpassant tous ses homologues dans une lutte pour le monopole de la définition légitime de tout ce que le nomos du système social concerné, le principe générateur de cette lutte (cf. supra), laisse dans le flou. Ainsi, dans le cas du champ littéraire, le mot « écrivain » ou le mot "écrire", par référence au nomos «Ici, on écrit (pour écrire)", "Ici, on est écrivain ${ }^{7}$ » (Bourdieu, 1992 : 298-321), sont l'enjeu de luttes permanentes de définition, à coups de réponses plus ou moins péremptoires à des questions anhistoriques telles que "qu'est-ce qu'écrire?», «qu'est-ce qu'écrire en tant qu'écrivain?», «par quels moyens? ", «à travers quels genres? ", «quels styles?», « dans quels buts? ", « pour qui ?», etc. - soit, comme le résumait Jean-Paul Sartre en 1948, "Qu'est-ce que la littérature?» (Sartre, 1948: 55-330). Or tous les efforts de réduction de ce flou, notamment les poétiques des écrivains, dominants et dominés, ont en commun de reposer sur la croyance qu'il existe quelque chose comme "la littérature ", qu'il vaut la peine de se mettre à son service et qu'elle a une valeur unique, inassimilable à quelque autre valeur. Cette unicité inestimable, cette exclusivité sans prix rend la valeur littéraire absolue, non seulement à nulle autre pareille, spécifiquement littéraire, mais vécue comme vitale. Valeur qui ne se résume donc pas, par exemple, à la valeur esthétique, avec laquelle on tend pourtant si souvent à la confondre, aussi bien sur le terrain de la production littéraire que dans le domaine de la critique littéraire (Denis, 2010 : §12).

11 Cette situation caractérise la littérature en langue française depuis que la France littéraire de Flaubert et de Baudelaire a atteint un degré d'indépendance politique, économique, religieuse, etc. tel qu'elle a pu s'engager dans une restructuration progressive en fonction de sa propre autonomie, qui n'avait fait que se renforcer en parallèle. "Autonomie » et "indépendance » ne désignent en effet pas la même chose : l'autonomie littéraire est la capacité à honorer soi-même (autos en grec) un principe fondateur (nomos) littéraire exclusif de toute contrainte d'une autre nature. Garantie par une indépendance obtenue de haute lutte contre les contraintes externes, cette autonomie est une manière de dépendance envers un mot d'ordre, mot d'ordre purement 
différentiel et ayant donc un caractère sacré (Dirkx, 2016a), sorte d'infinitif injonctif ${ }^{8}$, sans complément, intransitif : « écrire ».

Cet appel à la libido scribendi donne sens - orientation et signification - aux tours de passe-passe de la domination proprement littéraire. Aussi la reconnaissance littéraire n'est-elle pas un arbitraire pur : l'autonomie dont il vient d'être question et qui, en recul (Ducas, 2010), a néanmoins toujours valeur de modèle pour l'univers littéraire tout entier (Casanova, 1999), et non seulement l'autonomie mais tout le processus sociohistorique d'autonomisation qui s'accélère à « l'âge classique » (Viala, 1985 ; Bourdieu, 1992 : 75-164), est ce qui justifie petits et grands coups de force qui, ignorés comme tels, produisent la reconnaissance littéraire, puis la légitimité littéraire, ses hiérarchies et ses hiérarques. Les auteurs qui, situés au pôle autonome et détenteurs d'un capital symbolique suffisant, se trouvent en position d'universaliser leurs conceptions de la littérature, exercent une domination d'autant plus efficiente qu'ils le font en général, littéralement, de bonne foi, c'est-à-dire en vertu de ce que le nomos littéraire, qui imprègne toutes leurs pratiques comme celles de leurs pairs (potentiels), leur souffle à longueur de vie d'écrivain.

La reconnaissance varie donc selon le degré d'ajustement au nomos ou, plus précisément, selon "cette adhésion tacite au nomos, cette forme particulière de croyance » qu'est l' illusio, investissement dans (in) le jeu (ludus), «adhésion immédiate à la nécessité » du champ (Bourdieu, 1997 : 122). En tant que « croyance fondamentale dans la valeur des enjeux de la discussion et dans les présupposés inscrits dans le fait même de discuter ", l' illusio est « la condition indiscutée de la discussion » (Bourdieu, 1997 : 122). C'est elle qui explique que les débats littéraires soient non seulement animés, mais inégaux et hiérarchisés à l'aune d'échelles de normes discutables, au double sens de négociables et contestables. Tout le processus de reconnaissance repose in fine sur une injustice voilée par la magie de la croyance selon laquelle le nomos, véritable impératif catégorique, est fondamentalement juste et annule en les sublimant toutes les injustices.

\section{Reconnaissance littéraire et corps de l'écrivain}

Les études consacrées à la question axiologique en littérature auraient sans doute avantage à se pencher plus systématiquement sur les formes de la croyance spécifiquement littéraire, d'autant qu'elles-mêmes et leur objet plongent certaines de leurs racines dans le champ qui a fait de la croyance sa spécialité, à savoir la religion, et plus spécialement dans le sous-champ du christianisme, la principale des religions $d u$ texte sur le continent européen, berceau du texte littéraire.

Dans Les formes élémentaires de la vie religieuse, somme qu'il publie en 1912, Durkheim tente d'expliquer l'origine de la croyance religieuse (Durkheim, 2008). La thèse centrale, déjà énoncée quatorze ans plus tôt (Durkheim, 1996 : 1-48), en est la suivante : la société que forment les tribus primitives "est à ses membres ce qu'un dieu est à ses fidèles "; autrement dit, "le fidèle ne s'abuse pas quand il croit à l'existence d'une puissance morale dont il dépend et dont il tient le meilleur de lui-même : cette puissance existe, c'est la société » (Durkheim, 2008: 295 et 322). Ce que l'auteur appelle la «force religieuse » constitue selon lui la matrice historique de la «force impersonnelle » ou «force sociale ", c'est-à-dire une " énergie » issue des « représentations collectives » qui animent chaque membre d'une société et font de lui un être social et, par conséquent, un être humain (Durkheim, 2008 : 604-605). 
16 Mais on a moins vu que le corps qui, sous la plume de Durkheim, n'est guère plus qu'une « enveloppe matérielle » dans laquelle s'enracinent des représentations inférieures, car individuelles et d'origine biologique, occupe néanmoins une position-clef dans sa théorie. Sans pour autant l'ériger en objet de quelque sociologie du corps avant la lettre, Durkheim, un peu malgré lui, donne au corps le rôle d'une instance pivotale entre l'individuel et le social (Dirkx, 2016a). En effet, les représentations collectives ne peuvent exercer leur action vertueuse qu'en tant qu'elles sont «individualisées », « incarnées » par des corps. De même, les croyances ne peuvent se passer des pratiques rituelles collectives; ainsi, les célébrations de masse ne parviennent à maintenir la foi et par conséquent la cohésion de la tribu qu'à condition que chaque fidèle puisse s'extérioriser à travers des mouvements du corps: "C'est l'homogénéité de ces mouvements qui donne au groupe le sentiment de soi et qui, par conséquent, le fait être "(Durkheim, 2008 : 330).

17 Cela étant, Durkheim ne dit pas comment les représentations collectives viennent à l'individu, ni comment elles s'y maintiennent, si ce n'est par « les habitudes, les idées, les tendances que l'éducation a imprimées en nous " (Durkheim, 2008: 497). Une bonne partie de l'œuvre de Pierre Bourdieu a consisté à préciser cette pensée encore marquée par la tradition néoplatonicienne et néokantienne, et à substituer au corps-réceptacle un corps de part en part socialisé, y compris dans ses dimensions biologiques. Il n'existe aucune solution de continuité entre corps et âme, ni entre pratiques et croyances : «[L]es structures cognitives ne sont pas des formes de la conscience mais des dispositions du corps» (Bourdieu, 1997: 210). Ni conscientes, ni inconscientes, ces dispositions corporelles à l'évaluation et à l'action ainsi que leur organisation en ce que Bourdieu appelle un habitus sont le produit de l'assimilation de structures sociales par le corps d'un individu, le produit donc d'une socialisation qui marche à l'incorporation.

Incorporer signifie donc inscrire dans le corps, et dans la mesure où ce corps se vit ordinairement comme substance organique, intime, tout sauf sociale, les choses qui lui sont incorporées sont d'emblée naturalisées, vécues comme naturelles, comme évidentes. La socialisation ne cesse ainsi de générer des croyances, des évidences illusoires mais difficilement déracinables, car paraissant concorder - comme Durkheim l'avait bien vu à des réalités observables.

19 S'agissant de la socialisation dans ses dimensions littéraires, que nous appelons «littérarisation» (Dirkx, 2006: 28), celle-ci repose sur l'incorporation progressive de schèmes pratiques familiaux, scolaires, etc. sous forme de dispositions propres à être mobilisées dans la lecture ou dans l'écriture de type littéraire (littérarisation primaire), voire dans une activité au sein même du champ littéraire, en tant qu'écrivain, critique ou autre (littérarisation secondaire). À l'échelle d'un individu, la littérarisation produit un habitus littéraire ou sens pratique littéraire; à l'échelle d'un système littéraire, les dispositions produites par la littérarisation contribuent à reproduire dans une certaine mesure ce que véhiculent ses institutions, à savoir la doxa littéraire ou sens commun littéraire, le fonds commun d'évidences incorporées en matière de littérature (sous forme d'oppositions binaires entre réalité et fiction, texte et contexte, individu et société, auteur et narrateur, prose et poésie, etc.). C'est pourquoi le sens pratique littéraire et le sens commun littéraire, dont la rencontre fait, pour ainsi dire, jaillir les étincelles de l'écriture, gagnent à être analysés en prenant en compte leur enracinement dans des corps littérarisés.

20 Nous pouvons donc préciser maintenant que c'est l'ignorance ou l'oubli de l'incorporation qui est la condition d'efficacité des routines de la reconnaissance 
littéraire et de ses effets de pouvoir. La magie symbolique des récompenses littéraires n'opère qu'entre agents munis d'un corps et, de ce fait, disposés à méconnaître tout ce que cette magie symbolique doit au corps et tout ce que ce corps doit à la magie symbolique. Cela rend plus nécessaire encore l'étude conjointe de la croyance littéraire et de la croyance propre à la religion chrétienne, qui est non seulement la principale religion du texte concernée, mais la seule à être une religion de l'incarnation et, qui plus est, de l'ingestion du Père, de son incorporation dans un sens nouveau que Freud proposa pour la première fois un an après la parution des Formes élémentaires de la vie religieuse (Freud, 2015 : 180). Quoi qu'il en soit, les ressorts et les circuits de la reconnaissance littéraire s'avèrent étroitement liés aux fondements corporels de l'adhésion à la doxa littéraire, système d'évidences incorporées par les habitants d'un même univers littéraire et hiérarchisées autour de l'évidence fondatrice qu'est le nomos. Une meilleure compréhension de l'écriture littéraire et des ressorts de la reconnaissance qui l'animent suppose la prise en compte de l'écrivain dans sa réalité de corps littérarisé qui, à force de s'assimiler les phénomènes existants d'ordre littéraire, est toujours moins susceptible de les identifier pour ce qu'ils sont: des structures arbitraires, figées en institutions et en schèmes doxiques.

21 Alors qu'elle est généralement abordée à travers les modes de récompense institutionnels (prix, insertion dans les programmes scolaires, titre d'académicien, de directeur de collection, etc.), la question de la reconnaissance mériterait d'être étudiée plus souvent en termes de placements et de sens du placement, qui est un sens de la reconnaissance anticipée et une composante essentielle du sens pratique (Bourdieu, 1992: 363-364'). Ces sens s'originent en effet dans la structure d'intérêts singulière qu'est le champ littéraire. Plus précisément, ils sont inscrits dans chacun de ces prismes du champ que sont les corps, à l'intérieur desquels ils interagissent continuellement, motivant certains à se spécialiser dans la production de la reconnaissance (en tant que critiques ou éditeurs), d'autres dans sa «capitalisation » (en tant qu'auteurs), d'autres encore dans les deux à la fois. C'est que, comme tous les autres corps, le corps de l'écrivain a appris à écrire en étant sans cesse amené, dans les univers de contraintes familial et scolaire, à anticiper les sanctions positives et négatives de son écriture. Apprendre à écrire est se familiariser avec un système de reconnaissance. Placer un texte littéraire et se placer comme écrivain sont alors, du moins est-ce une hypothèse, deux investissements indissociables car indissociables du corps de l'écrivain, l'un dans le champ des prises de position, l'autre dans le champ des positions. L'écriture s'accompagne d'une constante anticipation, génératrice d'ajustements plus ou moins transgressifs ou autocensurants, des effets que le corps de l'écrivain sera susceptible de produire dans son texte et dans le champ, inséparablement champ de textes et champ de corps d'écrivains.

En régime d'autonomie, la plupart des écrivains inclinent à gommer ou à occulter leur corps. Au cœur de la doxa littéraire, il y a en effet l'image de l'écrivain comme instance beaucoup moins charnelle que spirituelle, beaucoup moins physique que symbolique, tel ce " prince des nuées » encombré par son corps « de géant » que met en scène, sous une forme allégorique, L'albatros de Baudelaire. Il faudrait analyser dans quelle mesure la reconnaissance d'un écrivain par ses pairs n'est pas d'abord reconnaissance tacite d'un corps fondamentalement silencieux, voire muet, s'effaçant devant la nécessité littéraire comme disait Samuel Beckett: "Bon qu'à ça ${ }^{10}$ " -, mais il faudrait citer aussi, entre beaucoup d'autres, les développements plus universalisants d'un Maurice Blanchot sur la mort de l'écrivain et sur le fait que, dans l'œuvre poétique, «personne ne parle[, car] la 
parole seule se parle » (Blanchot, $1984: 42$; cf. 203-206). Cela vaudrait la peine, dès lors que le nomos littéraire, "écrire", semble condamner le corps à se tenir en apesanteur sociale et historique, à se faire corps éthéré, décorporalisé, transsubstantié par l'écriture qui, seule, compte.

Mission impossible pour l'écrivain doté d'un corps réfractaire à pareille loi absolue mission impossible pour aucun écrivain, donc. D'autant moins pour ceux et celles qui ne correspondent pas au modèle de l'écrivain blanc, masculin et vivant de préférence en (Îlede-)France que continuent de propager la plupart des manuels, cours et programmes de recherche en littérature "française $»^{11}$. Cela dit, il y a corps d'écrivain et corps d'écrivain. Le Noir Dany Laferrière, de l'Académie française, a réussi à faire de nécessité vertu en faisant de son corps migrant la matière première de toute une œuvre, à commencer par son premier roman, sorte de mode d'emploi d'un genre nouveau expliquant Comment faire l'amour avec un nègre sans se fatiguer (Laferrière, 1985). Ce mode d'emploi est d'autant plus conforme sous le rapport de la littérature qu'il constitue en filigrane une longue déclaration d'amour du narrateur et romancier en herbe au nomos littéraire à travers sa relation avec "Mizz Littérature ", belle étudiante en Lettres, dont le grand corps est associé plus d'une fois à celui d'un albatros.

On voit ainsi que la littérature est un univers social où l'incorporation, l'enfouissement dans le corps des agents de schèmes pratiques, est plus créditée selon qu'elle implique l'enfouissement de ce corps en tant que tel. La reconnaissance n'advient qu'à mesure que le corps, y compris avec ses capacités "posturales" ou ses investissements " paratopiques ${ }^{12}$ ", se montre enclin non pas à produire, mais à subir la passion littéraire, soit comme l'« enveloppe matérielle » dont parlait Durkheim, caverne abritant quelque «moi intime», soit comme un corps-outil, canal médiumnique ou porte-plume. La reconnaissance littéraire reposerait ainsi sur la méconnaissance de ce qui la rend possible, c'est-à-dire non seulement la violence symbolique, mais le corps de l'écrivain tel qu'il est : littérarisé et, de ce fait (et non pas par magie), plus ou moins sujet à la passion littéraire et à la soumission au nomos. Cette passion étant ressentie comme une force qui transcende la passivité matérielle du corps, le monde littéraire comme champ relativement autonome récompense chaque écrivain en fonction (parmi d'autres compétences) de son aptitude à dominer son propre corps dans ses textes, à le sublimer, à le symboliser.

Prenons comme exemple le Baudelaire des Paradis artificiels. Celui-ci, tout comme bien d'autres avant et après lui, entendait mettre à l'épreuve son corps et lui faire subir des expériences spécifiquement littéraires visant à mesurer jusqu'à quel point le nomos littéraire - « la pure littérature » (Baudelaire, $2007: 232$ ) - pouvait avoir force de loi. Ces expériences faites, l'écriture devait lui permettre d'en rendre compte et, explique Claude Pichois, d'« introduire la mesure dans la démesure, tricher avec le dieu des paradis artificiels pour le rendre serf du poète »; écrire son livre, « d'abord, a été une libération : écrire sur le haschisch ou sur l'opium, c'est retrouver son autonomie d'être pensant " (Baudelaire, 2007: 20-21 et 19). Il nous semble que l'on peut ajouter à ces propos pertinents : « et c'est aussi, plus spécifiquement, retrouver son autonomie d'être pensant littéraire ", être d'un type nouveau, socialement inouï : l'écrivain autonome, libéré de tout et y compris, dans la mesure du possible, de son propre corps ${ }^{13}$.

26 Ainsi, une des figures-clefs de l'avènement d'un champ littéraire en France (Bourdieu, 1992: 93-103) recommande-t-elle à l'écrivain de symboliser son corps, y compris au moment même où il le met en scène dans son texte. C'est ce qu'exprime encore de nos 
jours, à l'heure du retour du «sujet» dans la littérature contemporaine française, Christine Angot, qui conteste sa réputation de romancière "autobiographique » ou «autofictionnelle» préconisant le tout dire à propos de son corps et de son corps d'écrivaine :

[...] c'est quand même un truc spécial, l'écriture. C'est une passion, vous n'avez pas le choix. C'est une question de désir, et contrairement à ce qu'on dit, le désir n'est pas quelque chose de... cool. Le désir, ça vous fait disparaître. Le « je » que j'emploie dans mes livres n'a rien à voir avec le « moi ». Il y a bien sûr des gens qui écrivent avec le moi qu'ils ont face à eux lorsqu'ils se regardent dans le miroir. C'est un travail d'écrivain possible, pourquoi pas, mais moi, ce n'est pas ce que je fais. Au contraire, je travaille à le faire disparaître, ce moi ${ }^{14}$.

L'incitation tacite du champ à «faire disparaitre » leur corps semble suivie par la plupart des écrivains, y compris par ceux qui donnent l'impression à une partie du public d'en faire étalage. Ainsi, le travail de symbolisation du corps doxique, ordinaire, c'est-à-dire littérairement insignifiant, apparaît à la fois comme un enjeu de taille et comme un instrument majeur de l'aspiration d'un auteur à être reconnu comme (grand) écrivain.

\section{Des corps complexes}

Selon sa littérarisation et selon sa position dans le champ (plus ou moins proche du pôle de l'autonomie et plus ou moins proche du pôle de la consécration littéraire), chaque écrivain effectue cet effacement de soi à sa manière. Les déterminants de l'écriture ne sont pas des déterminismes. Il serait même prématuré d'envisager l'hypothèse d'une corrélation entre le degré de reconnaissance et le degré de sublimation textuelle du corps de l'écrivain. Chacune de ces deux variables comporte en effet plusieurs facteurs et est elle-même prise dans un système de variables qu'il faudrait d'abord construire minutieusement : pour ce qui est de l'écriture du corps de l'écrivain, que nous proposons d'appeler « autographie », il s'agirait, entre autres, des modes de transposition d'éléments " autobiographiques ${ }^{15}$ ", des modalités de la narration (autodiégétique ou non), du rythme textuel (Meschonnic, 1982), des moyens de lexicalisation, de thématisation et d'exploration des corps (notamment du corps écrivant), etc. Toutes les hypothèses formulées dans les paragraphes précédents seraient à vérifier et nuancer à la lumière d'une étude différentielle de trajectoires représentatives d'un certain état de telle ou telle littérature, par exemple en langue française.

Nous nous contenterons d'esquisser ici, pour terminer, le cas de deux écrivains francophones qui ont progressivement mis en texte leur propre corps d'écrivain. Le premier, le Français Claude Simon ${ }^{16}$, n'en a vraisemblablement pas été disqualifié par le champ, ce qui ne semble pas avoir été le cas du second, le Belge Christian Dotremont.

La trajectoire de Claude Simon, né en 1913 et décédé en 2005, est marquée par des tensions de toutes sortes, génératrices de tiraillements de tous les instants. Ses origines sociales à la fois modestes du côté du père et élevées du côté de la mère, la mort prématurée de ses parents, sa scolarisation entamée tardivement, son rapport au travail en tant que rentier, etc., sont autant d'éléments déterminant ce qu'on peut appeler un habitus clivé. Cet habitus, on l'a vu, est le produit d'une incorporation de schèmes de vision de mondes différents, dont le premier, dans tous les sens du terme, est le monde familial ou plutôt, dans le cas de Claude Simon, les deux mondes familiaux fondés chacun sur un nomos exclusif de l'autre. Le caractère clivé de l'habitus de Claude Simon tient à la 
cohabitation en son corps d'appels mutuellement incompatibles (pour continuer l'exemple fondateur de la famille : appel du monde de la mère à s'engager sur une voie digne de son rang, contre appel paternel à une certaine modestie). Cette tension insoluble entre nomos par définition inconciliables et entre formes d'autonomie divergentes est ce qu'on peut appeler « antinomie » (Dirkx, 2011). L'habitus clivé, particulièrement empreint de dissonances antinomiques, tient à une socialisation structurée par la discordance entre deux modes de reproduction familiale, deux sortes de langue française, deux types de littérarisation, etc.

Or, l'antinomie interdit toute croyance paisible et donc toute pratique d'écriture apaisée. Ne reconnaissant aucun mot d'ordre si ce n'est celui de la perpétuation de l'écriture littéraire en dehors de tout modèle nécessairement restrictif, Claude Simon est livré à luimême et à sa propre propension à renouveler la tradition pour mieux la prolonger (Simon, 2006: 885-902). En lisant ses dix-sept romans, on assiste à un affrontement croissant entre certains dogmes romanesques et les audaces hérétiques d'un auteur au service exclusif d'une écriture pleinement littéraire. Inspiré par la peinture moderne, cet auteur entend réaliser un roman novateur (associé au "Nouveau Roman») qui soit principalement redevable aux logiques propres à l'écriture. Cette entreprise le conduit à préférer à l'évocation «réaliste » du monde les réminiscences désordonnées mais plus fiables que sa mémoire en fournit. Mais à donner ainsi libre cours à sa mémoire, son écriture toujours plus réflexive donne lieu progressivement à un retour du refoulé corporel dans son ensemble : les transgressions hétérodoxes favorisent et sont favorisées par l'apparition graduelle sur la scène diégétique des corps familiaux, puis des corps parentaux, puis de parties du corps de l'écrivain, lequel finit par sortir tout entier (et tout nu) des coulisses dans l'avant dernier roman, Le Jardin des Plantes (1997). La dialectique de la publication de l'œuvre et de sa reconnaissance (premières critiques), puis de sa légitimation (Prix de L'Express 1960, Prix Médicis 1967) et enfin de sa consécration venue de l'étranger (Prix Nobel 1985) s'assortit de l'émancipation, livre après livre, d'un corps de l'écrivain se sentant toujours plus légitimé à se désigner explicitement et librement comme source directe de la symbolisation littéraire, c'est-à-dire un corps se sentant toujours plus légitimé à (s')écrire.

La conjoncture littéraire était loin d'être aussi favorable au poète Christian Dotremont, qui compte également parmi les incarnations les plus achevées de l'autonomie littéraire en langue française de la seconde moitié $\mathrm{du} \mathrm{xx}^{\mathrm{e}}$ siècle (Dirkx, 2009). Sa trajectoire est tout aussi marquée par l'antinomie que celle de Claude Simon. Le village où il naît en 1922 et où il vivra jusqu'à sa mort en 1979, est situé à l'orée d'une grande forêt, à proximité de la capitale belge et au cœur d'une zone d'hybridation culturelle que l'on a coutume de réduire à une "frontière linguistique». Le couple parental constitue l'union des contraires sous plus d'un rapport (il finira par éclater). Quant à la littérarisation de Dotremont, elle est structurée par l'antinomie littéraire propre à la situation belge : habité par un mélange d'incertitude quant à ses spécificités et de surévaluation des modèles hexagonaux, tout écrivain belge francophone oscille entre, d'un côté, l'identification à ces modèles et à une « littérature française de Belgique » et, de l'autre côté, l'adhésion à une singularité locale et à une " littérature belge de langue française ». Il est ainsi tiraillé entre deux nomos littéraires qui, par définition, s'excluent mutuellement. Mais l'un étant plus légitime (« universel») que l'autre («régional»), l'antinomie littéraire tend à stimuler davantage la tentation d'incarner le modèle de 
l'« écrivain français ", y compris de la manière la plus totale, c'est-à-dire psychiquement et physiquement, en émigrant vers Paris.

Or, si le corps de Christian Dotremont est lui aussi sensible au chant des sirènes parisiennes dont le charme l'habite depuis toujours (il fera à Paris plusieurs longs séjours), tout le porte à rejeter les effets antinomiques de la domination littéraire française, vécus comme des contraintes hétéronomes. L'écrivain s'évertuera donc à les contourner, littéralement et littérairement, en se positionnant là où ses inclinations à la plus grande autonomie possible l'amèneront à se positionner. On le verra donc lancer un nouveau courant littéraire (le surréalisme révolutionnaire) dans l'espoir de subvertir le centre parisien par ses marges avant-gardistes. Viendra ensuite, en 1948, l'expérience de Cobra, conçue comme l'alliance des périphéries (COpenhague-BRuxelles-Amsterdam) et de leurs arts contre la capitale du monde artistique et intellectuel. Enfin, cette tentative ayant tourné court elle aussi, se trouvant seul et atteint d'une tuberculose contractée dans le froid du Danemark, son intransigeance l'éloignera de tous les sentiers battus pour le mener finalement dans les steppes enneigées de Laponie.

Dans la quasi-totalité des écrits de Dotremont, on retrouve cette hypersensibilité aux entre-deux, aux interstices, aux frontières entre l'ici et l'ailleurs, le rêve et le réel, l'animé et l'inanimé, le possible et l'impossible. En même temps s'y exprime une envie permanente de repousser ces frontières et de les dépasser dans un mouvement obstinément centrifuge et toujours plus enraciné dans la totalité du réel, donc toujours plus symbolique et physique. C'est dire que l'écrivain s'écarte toujours plus littéralement des circuits de la légitimation littéraire, de l'identification au statut d'écrivain arrivé qui, comme toute identification, lui paraît être un enfermement, mot qui est aussi à prendre dans sa dimension corporelle. Aussi les stratégies de placement recommandées par le champ ne sont-elles pour lui que méprisables «tactiques", mot récurrent sous la plume de cet écrivain toujours plus allergique à toute forme de légitimation de sa personne en lieu et place de son œuvre ${ }^{17}$. Même là où sa présence physique est exigée - et d'abord à Paris, pour une exposition personnelle ou une manifestation d'hommage -, il évitera le plus souvent de se montrer, préférant le va-et-vient entre son village natal et le Grand Nord. Mais c'est dans l'intimité de son grenier qu'il secouera, en 1962, un des derniers poids que la doxa littéraire fait peser sur l'écriture: celui de l'imprimerie, de la dactylographie, qui "tuent la moitié de l'écrivain, en tuant son écriture", car si «l'écrivain écrit, c'est d'abord dans le sens physique : avec la main ; c'est ensuite dans le sens "rédactionnel" » (Dotremont, $1998: 102)$. Il se lèvera donc de sa table d'écriture et se mettra à écrire ses poèmes en position debout, à même le sol, avec un pinceau : ce seront les « logogrammes ». À partir de l'année d'après, c'est à l'extrémité du monde habité, en Laponie, qu'il atteindra les limites du champ littéraire et de l'espace social, en allant y tracer, avec un bout de bois, d'éphémères « logoneiges » et « logoglaces ».

L'invention assez tardive par Dotremont, alors qu'il se savait condamné par Paris et par la maladie, de cet objet poétique et graphique qu'est le logogramme, déclencha sa légitimation, puis sa consécration posthume. De son côté, Claude Simon avait déjà parcouru, en partie sous l'égide du groupe du Nouveau Roman, la moitié du chemin vers l'émancipation de son corps d'écrivain, lorsque le champ littéraire international lui apporta la consécration et qu'il fut en position d'accomplir l'autre moitié dans ses derniers romans. Mais chacun de ces deux écrivains atteste à sa façon que la légitimité et la fertilité des luttes contre les conformismes littéraires se mesure aussi et peut-être 
surtout à la capacité de donner à lire l'écriture en tant qu'elle est l'expression d'un corps littérarisé, d'un être humain qui a appris à écrire littérairement.

\section{BIBLIOGRAPHIE}

BAUDELAIRE, Charles (2007). Les paradis artificiels [1860], précédé de La pipe d'opium, Le hachich, Le club des Hachichins par Théophile Gautier. Édition établie et présentée par Claude Pichois. Paris: Gallimard. BLANCHAUD, Corinne (dir.) (2015). Classique ou francophone? De la notion de classique appliquée aux œuvres francophones. Amiens: Encrage.

BLANCHOT, Maurice (1984). L'espace littéraire [1955]. Paris: Gallimard.

BOURDIEU, Pierre (1963). « La définition sociale de la photographie », in Pierre Bourdieu (dir.). Un art moyen. Essai sur les usages sociaux de la photographie. Paris: Minuit, pp. 107-138.

BOURDIEU, Pierre (1971). « Genèse et structure du champ religieux », Revue française de sociologie 12, 3 (juillet-septembre), pp. 295-334.

BOURDIEU, Pierre (1979). La distinction. Critique sociale du jugement. Paris: Minuit.

BOURDIEU, Pierre (1982). Ce que parler veut dire. L'économie des échanges linguistiques. Paris: Fayard. BOURDIEU, Pierre (1989). La noblesse d'État. Grandes écoles et esprit de corps. Paris: Minuit. BOURDIEU, Pierre (1992). Les règles de l'art. Genèse et structure du champ littéraire. Paris: Seuil. BOURDIEU, Pierre (1994). Raisons pratiques. Sur la théorie de l'action. Paris: Seuil. BOURDIEU, Pierre (1997). Méditations pascaliennes. Paris: Seuil. BOURDIEU, Pierre (2002). Questions de sociologie [1984]. Paris : Minuit. CASANOVA, Pascale (1999). La république mondiale des lettres. Paris: Seuil. DENIS, Benoît (2010). « La consécration. Quelques notes introductives », COnTEXTES 7. Approches de la consécration en littérature.

DIRKX, Paul (1993). « Y a-t-il des classiques belges? », Littératures Classiques 19 (automne 1993), pp. 313-327.

DIRKX, Paul (2006). Les « amis belges ». Presse littéraire et franco-universalisme. Rennes: Presses Universitaires de Rennes.

DIRKX, Paul (2009). «Christian Dotremont: l'ailleurs comme limite du champ littéraire », in Daniel Lançon et Patrick Née (dirs.), L'ailleurs depuis le romantisme. Essais sur les littératures en français. Paris: Hermann, pp. 303-328.

DIRKX, Paul (2011). «Claude Simon: antinomie et corps écrivant », in Paul Dirkx et Pascal Mougin (dirs.). Claude Simon: situations. Lyon: ENS Éditions, pp. 179-197.

DIRKX, Paul (2016a). «Le savant et le corps. Au seuil d'une théorie de l'incorporation : Les formes élémentaires de la vie religieuse d'Émile Durkheim », in Matthieu Béra, Nicolas Sembel (éds.). 
Durkheim et la religion. Les formes élémentaires de la vie religieuse cent ans après (1912-2012). Paris : Garnier [à paraître].

DIRKX, Paul (2016b). «Sacrée antinomie. L'engagement littéraire d'Ahmadou Kourouma », in Valentina Litvan (éd.). Littérature et sacré: la tradition en question. Actes du colloque international de Metz 21-22 novembre 2014. Bruxelles - Bern - Berlin - Francfort-sur-le-Main - New York - Oxford Vienne, PIE - Peter Lang [à paraître].

DOtRemont, Christian (1998). « Signification et sinification » [1950], Cobraland, Bruxelles: La Pierre d'Alun, pp. 99-102.

Dotremont, Christian, Butor, Michel (1986). Cartes et lettres. Correspondance 1966-1979. Établie et annotée par Michel Sicard. Paris : Galilée.

Dozo, Björn-Olav, Provenzano, François (2010). « Comment les écrivains sont consacrés en Belgique ", COnTEXTES 7. Approches de la consécration en littérature. <URL: https:// contextes.revues.org/4637>.

DUBoIs, Jacques (2012). « Devenir auteur: Angot actrice et témoin », in Björn-Olav Dozo, Anthony Glinoer, Michel Lacroix (éds.). Imaginaires de la vie littéraire. Fiction, figuration, configuration. Rennes: Presses Universitaires de Rennes, pp. 329-338.

DUCAS, Sylvie (2010). « Prix littéraires en France: consécration ou désacralisation de l'auteur? », COnTEXTES 7. Approches de la consécration en littérature. <URL: https://contextes.revues.org/4656> DURKHEIM, Émile (1996). « Représentations individuelles et représentations collectives », Revue de métaphysique et de morale 6, 3 (mai 1898), repris in id. Sociologie et philosophie. Paris : Presses Universitaires de France, pp. 1-48.

DURKHEIM, Émile (1997). Les règles de la méthode sociologique [1895]. Paris : Presses Universitaires de France.

DURKHEIM, Émile (2008). Les formes élémentaires de la vie religieuse. Le système totémique en Australie [1912]. Paris : Presses Universitaires de France.

ELIAS, Norbert (2015). Théorie des symboles [1991]. Traduit de l'anglais par Damien-Guillaume et MarieBlanche Audollent. Révisé, édité et présenté par Marc Joly. Paris : Seuil.

FREUD, Sigmund (2015). Totem et tabou [1913]. Paris : Presses Universitaires de France.

Kourouma, Ahmadou (1968). Les soleils des indépendances. Montréal : Presses de l'Université de Montréal.

LAFERRIÈRE, Dany (1985). Comment faire l'amour avec un nègre sans se fatiguer. Montréal, VLB. MESCHONNIC, Henri (1982). Critique du rythme. Anthropologie historique du langage. Lagrasse: Verdier. ROBERT, Lucie (2002). « Canon, canonisation », in Paul Aron, Denis Saint-Jacques, Alain Viala (dirs.). Le dictionnaire du littéraire. Paris: Presses Universitaires de France.

Sartre, Jean-Paul (1948). Situations II. Paris: Gallimard.

SCHIFFER, Daniel Salvatore (2008). Philosophie du dandysme. Une esthétique de l'âme et du corps (Kierkegaard, Wilde, Nietzsche, Baudelaire). Paris: Presses Universitaires de France.

SIMON, Claude (2006). CEuvres. Édition établie par Alastair B. Duncan, avec la collaboration de Jean $\mathrm{H}$. Duffy. Paris : Gallimard.

VIALA, Alain (1985). Naissance de l'écrivain. Sociologie de la littérature à l'âge classique. Paris : Minuit. VIALA, Alain (1993). Littératures Classiques 19 (automne 1993). Qu'est-ce qu'un classique? 


\section{NOTES}

1. Comme l'écrit Benoît Denis à propos de « consécration » (Denis, $2010:$ § 1).

2. Cette dialectique cognitive est une caractéristique fondamentale du mécanisme social de la reconnaissance, présente dans toute l'œuvre de Bourdieu (voir, par exemple, Bourdieu, 1994 :

187)

3. C'est nous qui soulignons. Ce lien, essentiel d'un point de vue heuristique, entre légitimation et domination passe souvent pour une évidence que l'on tend aussitôt à perdre de vue. Il n'est du reste pas rare que la notion de « domination » chez Bourdieu soit mésinterprétée ou euphémisée. 4. Notamment à la faveur de la diffusion en langue française, à partir des années 1990, de la théorie du polysystème d'Itamar Even-Zohar et Gideon Toury.

5. Voir le dossier Viala, 1993, et notamment, pour le cas belge, Dirkx, 1993. Sur la question des « classiques francophones ", lire Blanchaud, 2015.

6. Cf. Bourdieu, 1997: 123-127. Cette différence de degré - d'efficace et de publicité - passe souvent pour une différence de nature (p. ex. Denis, 2010 : § 12).

7. Par exemple, pour faire partie de l'Académie Goncourt, le testament d'Edmond de Goncourt stipule qu' «il sera nécessaire d'être homme de lettres, rien qu'homme de lettres, on n'y recevra ni grands seigneurs, ni hommes politiques » (Ducas, 2010 : § 7).

8. De type «Ne pas se pencher au-dehors». En français, l'infinitif exprimant un ordre est dit aussi " en modalité jussive ».

9. Pour une approche processuelle de la consécration des écrivains belges francophones, voir Dozo et Provenzano, 2010.

10. Libération mars 1985 (numéro hors série).

11. Voir plusieurs contributions in Blanchaud, 2015.

12. Nous pensons aux travaux de Jérôme Meizoz et de Dominique Maingueneau.

13. Pour de récents développements sur le dualisme corps-âme chez Baudelaire, on lira Schiffer, 2008.

14. Christine Angot in Nathalie Crom, "Christine Angot», Télérama 19 août 2015. Sur les ambivalences apparentes du désir d'autonomie littéraire d'Angot, voir Dubois, 2012.

15. "Le terme [autofiction] ressemble trop à "autobiographie". Je crains qu'une fois de plus on en déduise : «Ce n'est pas vraiment du roman" » (Christine Angot in « Angot, Millet : deux enquêtes sur l'amour ", Le Monde des livres 28 août 2008).

16. Nous n'avons jusqu'à présent trouvé aucun argument scientifique qui justifie d'écarter de l'étude des phénomènes littéraires francophones, c'est-à-dire en langue française, les textes produits par des écrivains français. La France littéraire est un élément, par ailleurs central, d'une configuration géolittéraire sans laquelle on ne saurait observer complètement aucun des autres éléments, ni la France littéraire elle-même.

17. Cet écrivain belge francophone est à mi-chemin entre la crispation identitaire et l'enchantement universaliste. Contrairement à la plupart de ses confrères «écrivains français de Belgique " publiant ou non en France, il ne chercha jamais à occulter ni sa nationalité, ni ses particularités langagières. Au contraire, il considéra toujours que c'est "la "culture française" [qui est] terriblement fermée » (Dotremont et Butor, 1986 : 132). 


\section{RÉSUMÉS}

Cette étude propose un ensemble d'hypothèses concernant les rapports entre la reconnaissance littéraire et les corps des écrivains. Une première partie tente de distinguer clairement, à travers une approche différentielle, les concepts de reconnaissance, notoriété, légitimité, légitimation, consécration, canonisation, classicisation et panthéonisation. Ce n'est qu'après ce nécessaire éclaircissement qu'on pourra étudier ce qui paraît être au fondement des mécanismes de reconnaissance littéraire: une autonomie elle-même basée sur une forme spécifiquement littéraire de la croyance. Cela amènera ensuite à convoquer la notion d'incorporation et à considérer les écrivains dans leurs dimensions inséparablement somatiques et symboliques. Enfin, certaines hypothèses seront nuancées à travers deux exemples d'écrivains qui ont mis en texte leur propre corps d'écrivain : Claude Simon et Christian Dotremont.

This study formulates a set of assumptions concerning the relations between literary recognition and the bodies of the writers. In a first part, a differential approach allows to make a clear distinction between the concepts of recognition, notoriety, legitimacy, legitimation, consecration, canonisation, classicisation and 'panthéonisation'. After this necessary clarification, it will be possible to look at what seems to be the foundation of literary recognition mechanisms: literary autonomy, based on a specifically literary form of belief. This will lead to introduce the concept of incorporation and to consider writers in both their somatic and symbolic dimensions, which are inseparably linked. Finally, some assumptions will be qualified through two examples of writers who have been putting into their texts their own writer's body: Claude Simon and Christian Dotremont.

\section{INDEX}

Mots-clés : reconnaissance, légitimation, autonomie, incorporation, corps de l'écrivain Keywords : recognition, legitimation, autonomy, incorporation, writer's body

\section{AUTEUR}

\section{PAUL DIRKX}

Un. de Lorraine

paul.dirkx[at]univ-lorraine.fr 